\title{
Medida da Cintura e Razão Cintura/Quadril e Identificação de Situaçōes de Risco Cardiovascular: Estudo Multicêntrico em Pacientes Com Diabetes Melito Tipo 2
}

\begin{abstract}
RESUMO
A obesidade abdominal está associada à presença de doenças cardiovasculares. O objetivo do presente trabalho foi avaliar, em pacientes com diabetes melito tipo 2 (DM2), o desempenho de duas medidas de obesidade abdominal, medida da circunferência da cintura e razão cintura/quadril (RCQ) na identificação de situações clínicas que representam risco cardiovascular: cardiopatia isquêmica, HAS, dislipidemia, obesidade e nefropatia diabética. Foi realizado um estudo transversal multicêntrico com 820 pacientes com DM2. A medida da cintura apresentou uma forte correlação com o índice de massa corporal (IMC), em homens $(r=0,814 ; P<0,05)$ e em mulheres $(r=0,770 ; P<0,05)$. Já a correlação da RCQ com o IMC foi fraca (homens: $r=0,263, P<0,05$; mulheres: $r=0,092, P<0,05)$. Apenas cintura se correlacionou com as medidas da pressão arterial sistólica $(r=0,211, P<$ 0,05 para homens; $r=0,224, P<0,05$ para mulheres). Na análise das áreas sob as curvas ROC, a cintura foi superior à RCQ para identificação de obesidade e HAS, tanto em homens como em mulheres, e para a dislipidemia em homens. Em conclusão, a circunferência da cintura está mais associada a situações de risco cardiovascular do que a RCQ. (Arq Bras Endocrinol Metab 2007;51/3:443-449)
\end{abstract}

Descritores: Obesidade abdominal; Circunferência da cintura; Razão cintura/quadril; Diabetes melito tipo 2

\section{ABSTRACT}

Waist Measure and Waist-to-Hip Ratio and Identification of Clinical Conditions of Cardiovascular Risk: Multicentric Study in Type 2 Diabetes Mellitus Patients.

Abdominal obesity is associated with cardiovascular disease. This study aims to compare two measures of abdominal obesity [waist and wais-tohip ratio (WHR)] in patients with DM2 to identify cardiovascular risk factors: ischemic cardiopathy, hypertension, dislipidemia, obesity and diabetic nephropathy. A multicentric study was performed in 820 patients with type 2 DM. Waist circumference strongly correlated with body mass index (BMI), for men $(r=0.814 ; P<0.05)$ and women $(r=0.770 ; P<0.05)$. On the other hand, WRH was weakly correlated $(r=0.263, P<0.05$ for men; $r=$ $0.092, P<0.05$ for women). Only waist circumference correlated with systolic pressure ( $r=0.211, P<0.05$ for men; $r=0,224, P<0.05$ for women). ROC curve analysis demonstrated the superiority of waist circumference measurement compared to WHR regarding obesity and hypertension for men and women, and dyslipidemia for men. In conclusion, waist circumference is better correlated with cardiovascular risk factor than WRH. (Arq Bras Endocrinol Metab 2007;51/3:443-449)

Keywords: Abdominal obesity; Waist circumference; Waist-to-hip ratio; Type 2 diabetes mellitus

\section{artigo original}

\author{
Paula Xavier Picon \\ Cristiane Bauerman Leitão \\ FERNANDO GERCHMAN \\ Mirela Jobim de AZEVEDO \\ SANDRa Pinho Silveiro \\ JORGE LUIZ GROSS \\ luís Henrique Canani
}

Programa de Pós-graduação em Ciências Médicas:

Endocrinologia e Metabologia, Universidade Federal do Rio Grande do Sul, Serviço de Endocrinologia do Hospital de Clínicas de Porto Alegre, RS.

Recebido em 31/05/06

Revisado em 05/10/06

Aceito em 15/10/06 
A o OBESIDADE CONTRIBUI PARA o desenvolvimento de várias doenças crônicas, incluindo a doença arterial coronariana, a hipertensão arterial sistêmica (HAS), a dislipidemia e o diabetes melito tipo 2 (DM2), levando a um maior risco de complicações cardiovasculares e morte (1). O índice de massa corporal (IMC) permite avaliar a obesidade e tem sido utilizado na estratificação de risco cardiovascular e nas decisões terapêuticas relacionadas às situações clínicas associadas. Entretanto, sabe-se, desde a década de 50 , que o tipo de distribuição de gordura corporal confere um risco variável de eventos cardiovasculares (2). A deposição excessiva de gordura visceral na região abdominal, chamada de obesidade androgênica, está associada a um risco maior de eventos coronarianos, DM2 e HAS, em ambos os sexos e em diferentes etnias (3-6). Acredita-se que esse aumento no risco de complicações vasculares deve-se à heterogeneidade das propriedades metabólicas e localização anatômica dos adipócitos, as quais levariam à resistência à ação da insulina (7).

Apesar de estar bem estabelecido que a gordura visceral abdominal faz parte da síndrome metabólica e está relacionada aos demais componentes da mesma (HAS, dislipidemia, alteração do metabolismo dos carboidratos), a melhor maneira de aferir o padrão de distribuição da gordura na prática clinica não está ainda definida. A quantificação acurada de tecido adiposo em compartimentos corporais pode ser realizada através de técnicas de imagem como ressonância magnética, tomografia computadorizada e densitometria corporal total (8). Entretanto, essas técnicas são onerosas e complexas, sendo impraticável sua utilização na rotina clínica. Já as medidas antropométricas simples, como a circunferência abdominal e a razão cintura/quadril (RCQ), demonstraram ser adequadas para estimar a quantidade de gordura abdominal. Recentemente, foi sugerido que a RCQ define melhor indivíduos de risco para doença cardiovascular (9-11). Outros autores consideram que a medida da cintura é melhor preditor de obesidade, dislipidemia e risco cardiovascular (4,12-14). Deve também ser considerado que o padrão de distribuição da gordura pode variar de acordo com os grupos étnicos, e tem sido recomendado que essas medidas sejam padronizadas para as diversas etnias (15-21). Ainda, os pontos de corte para a medida da cintura considerados como de risco cardiovascular, e que são utilizados no diagnóstico da síndrome metabólica, variam de acordo com as recomendações de diferentes sociedades $(21,22)$.

$\mathrm{O}$ objetivo do presente trabalho foi avaliar, em pacientes com DM2, o desempenho de duas medidas de obesidade abdominal, circunferência da cintura e RCQ, na identificação de situações clínicas que constituem risco cardiovascular: cardiopatia isquêmica, HAS, dislipidemia, obesidade e nefropatia diabética (ND).

\section{MATERIAL E MÉTODOS}

Foi realizado um estudo multicêntrico de teste diagnóstico no estado do Rio Grande do Sul, que incluiu 820 pacientes brancos com DM2 recrutados entre aqueles em atendimento ambulatorial no Serviço de Endocrinologia do Hospital de Clínicas de Porto Alegre $(n=448)$, no Hospital Nossa Senhora da Conceição $(n=192)$ e no Hospital São Vicente de Paula em Passo Fundo $(n=180)$. Definiu-se DM2 como valores de glicemia de jejum > 126 $\mathrm{mg} / \mathrm{dl}$, confirmados em pelo menos duas ocasiões, na ausência de episódios prévios de cetoacidose diabética, diagnóstico do diabetes melito após 30 anos de idade e ausência de necessidade de uso de insulina nos primeiros 5 anos de diagnóstico (23).

A avaliação dos pacientes seguiu um protocolo padronizado e previamente descrito (24), que incluiu dados demográficos, tempo conhecido de $\mathrm{DM}$, questionário cardiovascular da Organização Mundial de Saúde (OMS) (25) e avaliação da presença de ND. A pressão arterial sistêmica foi medida duas vezes após 10 minutos na posição sentada, no membro superior esquerdo, com esfigmomanômetro de coluna de mercúrio (fases I e V dos ruídos de Korotkoff). HAS foi definida pelas médias pressóricas $\geq 140 / 90 \mathrm{mmHg}$ ou pelo uso de medicações anti-hipertensivas. A medida de peso e altura foi feita em balança antropométrica sem sapatos e com roupas leves. O IMC foi calculado pela razão peso $(\mathrm{kg}) /$ altura $^{2}(\mathrm{~m})$. Obesidade foi definida pelo IMC $\geq 30$ $\mathrm{kg} / \mathrm{m}^{2}$. Foram medidas a circunferência da cintura (na altura da cicatriz umbilical) e do quadril (porção de maior diâmetro) e foi calculada a RCQ. A ND foi definida por aumento na excreção urinária de albumina (EUA) em urina de $24 \mathrm{~h}$ minutada ou amostra casual de urina $(>20 \mu \mathrm{g} / \mathrm{min}$ ou $17 \mathrm{mg} / \mathrm{l}$ ) na ausência de infecção urinária ou outras anormalidades renais e sem uso de inibidores da enzima conversora. A ND foi classificada em microalbuminúria (EUA 20-199 $\mu \mathrm{g} / \mathrm{min}$ ou $17-173 \mathrm{mg} / \mathrm{l}$ ) ou macroalbuminúria (EUA $\geq 200 \mu \mathrm{g} / \mathrm{min}$ ou $\geq 174 \mathrm{mg} / \mathrm{l}$ ), de acordo com a EUA em urina de $24 \mathrm{~h}$ ou em amostra, respectivamente $(26,27)$. O diagnóstico de micro ou macroalbuminúria foi confirmado em pelo menos uma segunda medida isolada.

A dislipidemia foi definida como sugerido pelo NCEP (22) no diagnóstico da síndrome metabólica: triglicerídeos séricos $\geq 150 \mathrm{mg} / \mathrm{dl}$ ou $\mathrm{HDL}<40 \mathrm{mg} / \mathrm{dl} \mathrm{em}$ homens e $<50 \mathrm{mg} / \mathrm{dl} \mathrm{em} \mathrm{mulheres.}$

Cardiopatia isquêmica foi diagnosticada na presença de angina ou possível infarto de acordo com questionário cardiovascular da OMS (25), e/ou de alterações eletrocardiográficas (código Minnesota) e/ou de anormalidades perfusionais (fixas ou variáveis) na cintilografia miocárdica em repouso e após dipiridamol. 


\section{Avaliaçāo laboratorial}

A albuminúria foi medida através da técnica de imunoturbidimetria [MicroAlb SeraPak (Bayer, Tarrytown, NY]. Os parâmetros metabólicos foram analisados pela manhã após 12 horas de jejum. A glicose plasmática foi determinada pelo método glicose oxidase, teste Alc por HPLC - (valores de referência: 4,7-6,0\%; Merck, Damstadt, Germany); triglicerídeos e níveis de colesterol por método enzimático. LDL-colesterol foi calculado pela equação de Friedewald. A insulina sérica foi dosada por radioimunoensaio (Elecsys $\mathrm{R}$ Systems 1010 / 2010 / análise modular E170 - ROCHE). A resistência insulínica foi estimada através do cálculo do Homeostasis Model Assessment [HOMA-IR= insulina em jejum $(\mathrm{pmol} / \mathrm{l}) \mathrm{x}$ glicose plasmática em jejum $(\mathrm{mmol} / \mathrm{l}) /$ $22,5]$. Para conversão dos valores de glicose em $\mathrm{mg} / \mathrm{dl}$ para $\mathrm{mmol} / \mathrm{l}$, foi utilizado o fator $0,0555 \mathrm{l}$.

\section{Consideraçōes éticas}

O protocolo foi aprovado pelo Comitê de Ética em Pesquisa dos centros participantes, e todos pacientes assinaram o termo de consentimento livre e esclarecido.

\section{Análise estatística}

Coeficientes de correlação de Spearman foram utilizados para avaliar as correlações entre as medidas da cintura e da RCQ com as variáveis contínuas em estudo.

A construção de curvas ROC foi utilizada para avaliar o desempenho das medidas da cintura e da RCQ na identificação de desfechos clínicos de interesse: cardiopatia isquêmica, HAS, dislipidemia, obesidade e ND. A área estimada sob cada curva varia de 0,5 (ausência de acurácia) a 1,0 (acurácia máxima). As curvas foram expressas pela estimativa pontual da área e estimativa do intervalo de confiança (IC) de 95\%. Curvas com áreas > 0,5 são consideradas úteis na identificação de situações de interesse. Curvas com áreas cujo intervalo de confiança inclui o 0,5 não adicionam informação (28). As curvas ROC foram também utilizadas para avaliar a especificidade e a sensibilidade dos pontos de corte para medida da cintura propostos pelo National Cholesterol Education Program(NCEP) (102 cm para homens e $88 \mathrm{~cm}$ para mulheres) eInternational Diabetes Federation (IDF) (94 cm para homens europeus e 80 $\mathrm{cm}$ para mulheres européias) $(21,22)$. Todas as análises foram feitas em separado para homens e mulheres.

Os dados foram descritos como média \pm desviopadrão ou números absolutos e percentuais. As variáveis com distribuição não-normal sofreram transformação logarítmica. O critério de alfa de $5 \%$ foi considerado significante. As análises foram realizadas no programa Statistical Package for the Social Sciences (SPSS, versão 10.0 para Windows).

\section{RESULTADOS}

Um total de 820 pacientes brancos com DM2 (378 homens, 442 mulheres) foi incluído no estudo. Os pacientes apresentaram $57,5 \pm 10,3$ anos de idade e duração do DM de $13,1 \pm 5,6$ anos. O valor de colesterol total foi $210 \pm 45 \mathrm{mg} / \mathrm{dl}$, colesterol HDL de 46 $\pm 12 \mathrm{mg} / \mathrm{dl}$, colesterol LDL $145 \pm 10 \mathrm{mg} / \mathrm{dl} \mathrm{e}$ triglicerídeos $145 \mathrm{mg} / \mathrm{dl}$ (27 a 1237). Quanto aos parâmetros antropométricos, o IMC foi $29,6 \pm 6,0$ $\left(\mathrm{kg} / \mathrm{m}^{2}\right)$ para mulheres e $28,1 \pm 4,7\left(\mathrm{~kg} / \mathrm{m}^{2}\right)$ para homens, a RCQ foi $0,93 \pm 0,07$ para mulheres e 0,98 $\pm 0,06$ para homens e a cintura foi $96,9 \pm 12,0 \mathrm{~cm}$ para mulheres e 99,4 $\pm 11,7 \mathrm{~cm}$ para homens.

\section{Correlaçōes}

$\mathrm{Na}$ tabela $\mathrm{l}$ estão descritas as correlações entre a RCQ e a medida da cintura com variáveis que estimam fatores de risco cardiovasculares. A medida da cintura e a RCQ se correlacionaram com o IMC, sendo que a cintura apresentou uma forte correlação, tanto para homens $(\mathrm{r}=0,814 ; P<0,001)$ quanto para mulheres $(\mathrm{r}=0,770 ; P<0,001)$, enquanto que a RCQ apresentou uma fraca correlação $(\mathrm{r}=0,263, P<0,001$ para homens; $r=0,092, P<0,044$ para mulheres) (figura 1 ). $\mathrm{O}$ mesmo foi observado para o peso. Para cada aumento de um quilo no peso corporal observou-se um aumento na medida da cintura de 0,69 $\mathrm{cm}$ nas mulheres e $0,63 \mathrm{~cm}$ nos homens. Apenas a cintura se correlacionou com as medidas da pressão arterial sistêmica tanto nos homens como nas mulheres. A correlação das duas medidas de obesidade abdominal foi semelhante em relação aos níveis séricos de triglicerídeos $(P<0,05)$. Não se observou correlação significativa dessas medidas de obesidade abdominal com os níveis séricos de teste $\mathrm{Alc}$, colesterol total, colesterol HDL, colesterol LDL, albuminúria e HOMA-IR.

\section{Análise de curva ROC}

Os valores da área sob a curva para identificação de situações clínicas de risco cardiovascular através da medida da cintura e da RCQ estão expressos na tabela 2.

Em homens, tanto a medida da cintura quanto a RCQ foram úteis na identificação de HAS e de obesidade (áreas $>0,5$ ). Também nos homens, a cintura se mostrou útil na identificação de dislipidemia, enquanto a RCQ não adicionou informação. Nas mulheres, a medida da cintura foi útil na identificação da HAS e da obesidade, enquanto a medida da RCQ foi útil somente na identificação da dislipidemia, não sendo superior à medida isolada da cintura.

Nem a cintura nem a RCQ foram úteis para identificação da presença de cardiopatia isquêmica e $\mathrm{ND}$, tanto em homens quanto em mulheres, pois as áreas das curvas incluíram o valor 0,5 .

A sensibilidade e a especificidade dos pontos de corte da medida da cintura sugeridos pelo NCEP e 
Tabela 1. Correlação entre a razão cintura-quadril e a medida da circunferência da cintura com variáveis que estimam fatores de risco cardiovasculares.

\begin{tabular}{|c|c|c|c|c|}
\hline & \multicolumn{2}{|c|}{ Homens } & \multicolumn{2}{|c|}{ Mulheres } \\
\hline & RCQ & Cintura & RCQ & Cintura \\
\hline IMC (kg/m²) & $0,263^{*}$ & $0,814 *$ & $0,092 *$ & $0,770 *$ \\
\hline Peso $(\mathrm{kg})$ & $0,108 *$ & $0,806 *$ & $0,296 *$ & $0,819 *$ \\
\hline Pressão sistólica (mmHg) & 0,055 & $0,211 *$ & $-0,009$ & $0,224 *$ \\
\hline Pressão diastólica $(\mathrm{mmHg})$ & 0,063 & $0,235 *$ & $-0,056$ & $0,268 *$ \\
\hline Teste A1c (\%) & 0,031 & $-0,058$ & 0,017 & $0,198^{*}$ \\
\hline HOMA-IR* & 0,062 & 0,139 & 0,025 & 0,194 \\
\hline Colesterol total (mg/dl)I & 0,040 & 0,027 & 0,120 & 0,077 \\
\hline Triglicerídeos (mg/dl) & $0,228 *$ & $0,200 *$ & $0,163^{*}$ & $0,138^{*}$ \\
\hline HDL colesterol (mg/dl) & $-0,012$ & $-0,089$ & $-0,029$ & $-0,079$ \\
\hline LDL colesterol (mg/dl) & 0,002 & 0,102 & 0,122 & 0,061 \\
\hline Albuminúria ( $\mu \mathrm{g} / \mathrm{min})$ & 0,048 & 0,085 & 0,043 & 0,002 \\
\hline
\end{tabular}

\section{Mulheres}
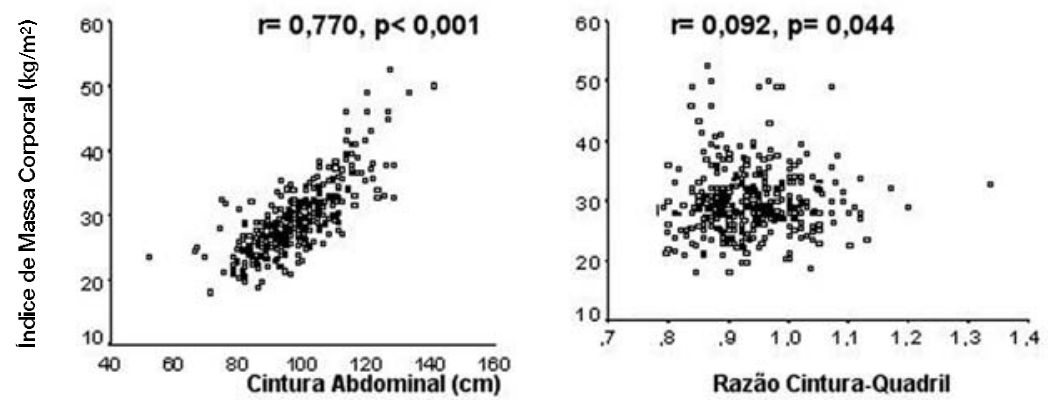

Homens
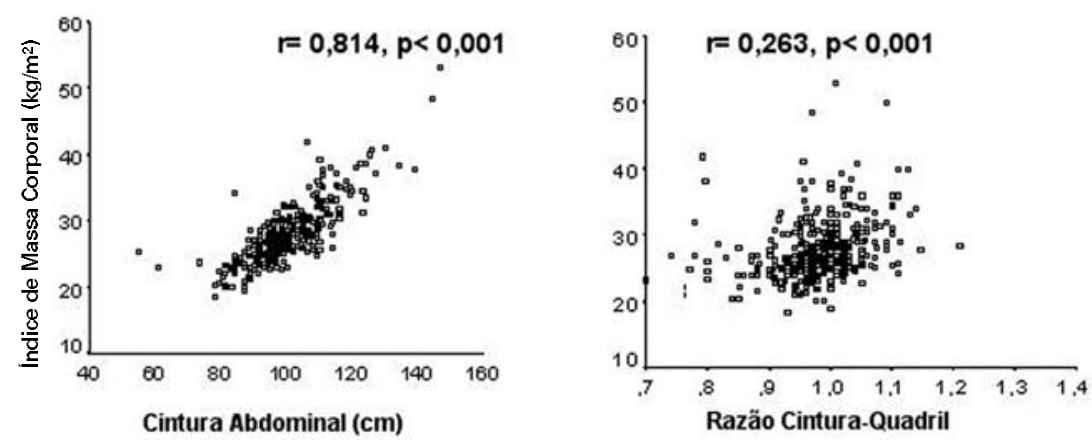

Figura 1. Correlações entre o índice da massa corporal e cintura abdominal e razão cintura-quadril entre homens e mulheres.

pelo IDF na identificação dos desfechos clínicos de interesse estão descritas na tabela 3 . O ponto de corte sugerido pelo NCEP para homens $(102 \mathrm{~cm})$ apresentou elevada especificidade e baixa sensibilidade e para as mulheres $(88 \mathrm{~cm})$, elevada sensibilidade e baixa especificidade. Já os pontos de corte das medidas de cintura adotados pelo IDF apresentaram elevada sensibilidade e baixa especificidade tanto para homens como para mulheres em relação à identificação dos desfechos clínicos avaliados.

\section{DISCUSSĀO}

A presente análise mostrou que o uso da cintura isoladamente foi superior ao uso da RCQ na identificação de algumas situações clínicas de risco cardiovascular em pacientes com DM2. Em homens e mulheres, a medida da cintura foi útil na identificação da HAS, da dislipidemia e da obesidade.

Embora a RCQ possa ser considerada a técnica antropométrica tradicional para verificar obesidade 
Tabela 2. Valores das áreas sob as curvas ROC para a medida da cintura e da RCO na identificação de situações clínicas de risco cardiovascular.

\begin{tabular}{lcccc}
\hline & \multicolumn{2}{c}{ RCQ } & \multicolumn{2}{c}{ Cintura } \\
\hline Desfecho Clínico & Área & IC 95\% & Área & IC 95\% \\
Homens & & & & \\
Cardiopatia isquêmica & 0,587 & $0,487-0,686$ & 0,506 & $0,399-0,613$ \\
\hline Nefropatia & 0,587 & $0,499-0,675$ & 0,582 & $0,495-0,670$ \\
Retinopatia & 0,521 & $0,422-0,620$ & 0,495 & $0,398-0,592$ \\
HAS & 0,603 & $0,513-0,693$ & 0,658 & $0,574-0,742$ \\
\hline Dislipidemia & 0,555 & $0,466-0,643$ & 0,608 & $0,519-0,697$ \\
\hline Obesidade & 0,647 & $0,548-0,745$ & 0,917 & $0,873-0,961$ \\
Mulheres & & & & \\
Cardiopatia isquêmica & 0,561 & $0,463-0,658$ & 0,511 & $0,417-0,607$ \\
\hline Nefropatia & 0,428 & $0,344-0,512$ & 0,527 & $0,440-0,615$ \\
\hline Retinopatia & 0,576 & $0,490-0,661$ & 0,541 & $0,454-0,628$ \\
\hline HAS & 0,511 & $0,420-0,602$ & 0,714 & $0,637-0,791$ \\
\hline Dislipidemia & 0,583 & $0,505-0,661$ & 0,587 & $0,509-0,665$ \\
\hline Obesidade & 0,559 & $0,479-0,639$ & 0,854 & $0,873-0,961$ \\
\hline
\end{tabular}

Dados expressos em áreas sob a curva (intervalo de confiança).

$\mathrm{RCQ}=$ razão cintura/quadril; $\mathrm{ROC}=$ Receiver Operator Characteristic; $\mathrm{HAS}=$ hipertensão arterial sistêmica; IC $95 \%=$ intervalo de confiança de $95 \%$

Tabela 3. Valores de sensibilidade e especificidade das medidas de cintura propostos pelo NCEP* e IDF** para a identificação das situações clínicas de risco cardiovascular.

\begin{tabular}{|c|c|c|c|}
\hline & & Sensibilidade & Especificidade \\
\hline \multicolumn{4}{|l|}{ Homens } \\
\hline \multirow[t]{2}{*}{ Cardiopatia isquêmica } & NCEP & $33 \%$ & $75 \%$ \\
\hline & IDF & $62 \%$ & $28 \%$ \\
\hline \multirow[t]{2}{*}{ Nefropatia diabética } & NCEP & $39 \%$ & $73 \%$ \\
\hline & IDF & $75 \%$ & $37 \%$ \\
\hline \multirow[t]{2}{*}{ HAS } & NCEP & $37 \%$ & $80 \%$ \\
\hline & IDF & $69 \%$ & $40 \%$ \\
\hline \multirow[t]{2}{*}{ Dislipidemia } & NCEP & $35 \%$ & $72 \%$ \\
\hline & IDF & $71 \%$ & $43 \%$ \\
\hline \multirow[t]{2}{*}{ Obesidade } & NCEP & $82 \%$ & $82 \%$ \\
\hline & IDF & $100 \%$ & $45 \%$ \\
\hline \multicolumn{4}{|l|}{ Mulheres } \\
\hline \multirow[t]{2}{*}{ Cardiopatia isquêmica } & NCEP & $72 \%$ & $30 \%$ \\
\hline & IDF & $95 \%$ & $10 \%$ \\
\hline \multirow[t]{2}{*}{ Nefropatia diabética } & NCEP & $74 \%$ & $26 \%$ \\
\hline & IDF & $98 \%$ & $6 \%$ \\
\hline \multirow[t]{2}{*}{ HAS } & NCEP & $78 \%$ & $45 \%$ \\
\hline & IDF & $92 \%$ & $8 \%$ \\
\hline \multirow[t]{2}{*}{ Dislipidemia } & NCEP & $79 \%$ & $34 \%$ \\
\hline & IDF & $94 \%$ & $10 \%$ \\
\hline \multirow[t]{2}{*}{ Obesidade } & NCEP & $94 \%$ & $39 \%$ \\
\hline & IDF & $95 \%$ & $10 \%$ \\
\hline
\end{tabular}

*NCEP $=$ National Cholesterol Education Program; ${ }^{*}$ IDF= International Diabetes Fede ration; $\mathrm{HAS}=$ hipertensão arterial sistêmica

Ponto de corte para medida da circunferência da cintura: NCEP homens: $102 \mathrm{~cm}$, muIheres: $88 \mathrm{~cm}$; IDF homens: $94 \mathrm{~cm}$, mulheres: $80 \mathrm{~cm}$.

central, o uso da circunferência da cintura tem ganhado suporte como uma opção mais simples $(4,29,30)$. O uso de uma variável única reduz a chance de erro e, além disso, o tamanho do quadril está fortemente associado com a estrutura da pelve (31). A circunferência da cintura pode ser um indicador antropométrico de gordura abdominal, pois parece ser menos afetada pelo sexo ou grau de obesidade total (30).
Contrários ao uso da medida da cintura como medida de obesidade abdominal, alguns investigadores têm relatado que a RCQ seria um melhor preditor de fatores de risco cardiovasculares. Em um estudo de base populacional finlandês, que avaliou prospectivamente 1.346 indivíduos masculinos por até 14 anos, a RCQ, a medida da cintura e o IMC foram diretamente associados ao desenvolvimento de eventos coronarianos. Homens com RCQ no quartil superior $(>0,91)$ 
tiveram um risco de eventos coronarianos aumentado em três vezes, enquanto os homens com a cintura no quartil superior $(90 \mathrm{~cm})$ tiveram um aumento de duas vezes. Neste estudo, a obesidade abdominal foi mais importante que a obesidade total, e a inclusão do IMC no modelo estatístico não adicionou informação à medida da RCQ isolada (32). Por outro lado, a concentração sérica de leptina está mais fortemente associada com a circunferência da cintura do que com a RCQ, em ambos os sexos (33). Isso pode ser devido, em parte, à maior associação observada entre o IMC com a circunferência da cintura do que com a RCQ (34), como foi também demonstrado no presente estudo.

A utilização do ponto de corte proposto pelo NCEP nos pacientes brancos com DM2 apresentou baixa sensibilidade entre os homens na identificação das diversas situações de risco cardiovascular, sugerindo que um número grande de indivíduos não seria identificado por este critério. Já entre as mulheres, o ponto sugerido pelo NCEP apresentou maior sensibilidade, com baixa especificidade, sugerindo que a utilização desse critério evitaria a perda de mulheres com as situações de risco cardiovascular às custas de elevado número de falso-positivos. Esses dados sugerem que novos pontos de corte para definição de valores de circunferência de cintura que possam ser positivamente associados à risco cardiovascular devem ser definidos para a população branca brasileira. Neste sentido, os valores sugeridos pelo IDF, por apresentarem uma maior sensibilidade, permitiriam que um maior número de pacientes considerados de risco não deixassem de ser analisados e/ou acompanhados em relação a situações de risco cardiovascular.

Uma das possíveis limitações deste estudo é o seu delineamento transversal, que permite apenas analisar a associação dos dados. Estudos prospectivos de longo prazo com desfechos definitivos (por exemplo, infarto agudo do miocárdio, insuficiência renal crônica, morte) permitiriam a inferência de uma relação causa-efeito. Entretanto, esses estudos são caros, complexos e ainda não disponíveis. Por outro lado, o grande número de pacientes incluídos no presente estudo reforça a importância dos achados descritos.

Em conclusão, a medida da circunferência da cintura é um bom parâmetro para a avaliação de obesidade abdominal em brasileiros brancos com DM2, podendo ser um dado clínico adicional para a identificação de situações clínicas que representem risco cardiovascular aumentado. Estudos prospectivos, incluindo outras etnias brasileiras, são necessários para corroborar esses achados.

\section{AGRADECIMENTOS}

Este estudo foi parcialmente financiado pelo Programa de Apoio a Núcleos de Excelência, Coordenação de Aperfeiçoamento de Pessoal de Nível Superior (CAPES), Conselho Nacional de Desenvolvimento Científico e Tecnológico (CNPq) e Hospital de Clínicas de Porto Alegre.

\section{REFERÊNCIAS}

1. Karelis AD, St Pierre DH, Conus F, Rabasa-Lhoret $R$, Poehlman ET. Metabolic and body composition factors in subgroups of obesity: what do we know? J Clin Endocrinol Metab 2004;89(6):2569-75.

2. Vague J. [Obesity in the development of arteriosclerosis and diabetes.]. Sem Hop 1954;30(58):3244-6.

3. Cabrera MA, Wajngarten M, Gebara OC, Diament J. [Relationship between body mass index, waist circumference, and waist-to-hip ratio and mortality in elderly women: a 5-year follow-up study]. Cad Saúde Pública 2005;21(3):767-75.

4. Han TS, van Leer EM, Seidell JC, Lean ME. Waist circumference action levels in the identification of cardiovascular risk factors: prevalence study in a random sample. BMJ 1995;311(7017):1401-5.

5. Olinto MT, Nacul LC, Gigante DP, Costa JS, Menezes AM, Macedo S. Waist circumference as a determinant of hypertension and diabetes in Brazilian women: a population-based study. Public Health Nutr 2004;7(5):629-35.

6. Nicklas BJ, Penninx BW, Cesari M, Kritchevsky SB, Newman $A B$, Kanaya AM, et al. Association of visceral adipose tissue with incident myocardial infarction in older men and women: the Health, Aging and Body Composition Study. Am J Epidemiol 2004;160(8):741-9.

7. Bray GA. Medical consequences of obesity. J Clin Endocrinol Metab 2004;89(6):2583-9.

8. Garg A. Regional adiposity and insulin resistance. J Clin Endocrinol Metab 2004;89(9):4206-10.

9. Kragelund C, Omland T. A farewell to body-mass index? Lancet 2005;366(9497):1589-91.

10. Dalton M, Cameron AJ, Zimmet PZ, Shaw JE, Jolley D, Dunstan DW, et al. Waist circumference, waist-hip ratio and body mass index and their correlation with cardiovascular disease risk factors in Australian adults. J Intern Med 2003;254(6):555-63.

11. Esmaillzadeh A, Mirmiran P, Azizi F. Waist-to-hip ratio is a better screening measure for cardiovascular risk factors than other anthropometric indicators in Tehranian adult men. Int J Obes Relat Metab Disord 2004;28(10):1325-32.

12. Lemos-Santos MG, Valente JG, Gonçalves-Silva RM, Sichieri R. Waist circumference and waist-to-hip ratio as predictors of serum concentration of lipids in Brazilian men. Nutrition 2004;20(10):857-62.

13. Neovius $M$, Linne $Y$, Rossner $S$. BMI, waist-circumference and waist-hip-ratio as diagnostic tests for fatness in adolescents. Int J Obes (Lond) 2005;29(2):163-9.

14. Wang Z, Hoy WE. Waist circumference, body mass index, hip circumference and waist-to-hip ratio as predictors of cardiovascular disease in Aboriginal people. Eur J Clin Nutr 2004;58(6):888-93.

15. Misra A, Vikram NK, Gupta R, Pandey RM, Wasir JS, Gupta VP. Waist circumference cutoff points and action levels for Asian Indians for identification of abdominal obesity. Int J Obes (Lond) 2006;30(1):106-11.

16. Wildman RP, Gu D, Reynolds K, Duan X, He J. Appropriate body mass index and waist circumference cutoffs for categorization of overweight and central adiposity among Chinese adults. Am J Clin Nutr 2004;80(5):1129-36. 
17. Zhou BF, Wu YF, Li Y, Zhang LF. [The cut-off point of waist circumference for identifying metabolic syndrome in Chinese adults]. Zhonghua Xin Xue Guan Bing Za Zhi 2005;33(1):81-5.

18. Tan CE, Ma S, Wai D, Chew SK, Tai ES. Can we apply the National Cholesterol Education Program Adult Treatment Panel definition of the metabolic syndrome to Asians? Diabetes Care 2004;27(5):1182-6.

19. Zhu S, Heymsfield SB, Toyoshima H, Wang Z, Pietrobelli A, Heshka S. Race-ethnicity-specific waist circumference cutoffs for identifying cardiovascular disease risk factors. Am J Clin Nutr 2005;81(2):409-15.

20. Rosenbaum P, Gimeno SG, Sanudo A, Franco LJ, Ferreira SR. Analysis of criteria for metabolic syndrome in a populationbased study of Japanese-Brazilians. Diabetes Obes Metab 2005;7(4):352-9.

21. International Diabetes Federation. The IDF consensus worldwide definition of the metabolic syndrome. Available at: <http://www.idf.org>. Accessed in 2005.

22. Executive Summary of The Third Report of The National Cholesterol Education Program (NCEP) Expert Panel on Detection, Evaluation, And Treatment of High Blood Cholesterol In Adults (Adult Treatment Panel III). JAMA $2001 ; 285(19): 2486-97$.

23. Report of the Expert Committee on the Diagnosis and Classification of Diabetes Mellitus. Diabetes Care 1997;20(7): 1183-97.

24. Scheffel RS, Bortolanza D, Weber CS, Costa LA, Canani LH, Santos KG, et al. Prevalência de complicações micro e macrovasculares e de seus fatores de risco em pacientes com diabetes melito do tipo $2 \mathrm{em}$ atendimento ambulatorial. Rev Assoc Med Bras 2004;50(3):263-7.

25. Coronary Drug Project Research Group. Circulation 1973;48(suppl. 1):47-8.

26. Zelmanovitz T, Gross JL, Oliveira JR, Paggi A, Tatsch M, Azevedo MJ. The receiver operating characteristics curve in the evaluation of a random urine specimen as a screening test for diabetic nephropathy. Diabetes Care 1997; 20 (4):516-9.
27. Costa LA, Canani LH, Maia AL, Gross JL. The ACE insertion/deletion polymorphism is not associated with the metabolic syndrome (WHO Definition) in Brazilian type 2 diabetic patients. Diabetes Care 2002;25(12):2365-6.

8. Medcalc Manual. Version 8.1.1.0, 2005.

29. Lean ME, Han TS, Morrison CE. Waist circumference as a measure for indicating need for weight management. BMJ 1995;311(6998):158-61.

30. Lemieux S, Prud'homme D, Bouchard C, Tremblay A Despres JP. A single threshold value of waist girth identifies normal-weight and overweight subjects with excess visceral adipose tissue. Am J Clin Nutr 1996;64(5):685-93.

31. Ley CJ, Lees B, Stevenson JC. Sex- and menopause-associated changes in body-fat distribution. Am J Clin Nutr 1992;55(5):950-4.

32. Lakka HM, Lakka TA, Tuomilehto J, Salonen JT. Abdominal obesity is associated with increased risk of acute coronary events in men. Eur Heart J 2002;23(9):706-13.

33. Zimmet $P$, Hodge A, Nicolson M, Staten M, de Court, Moore $J$, et al. Serum leptin concentration, obesity, and insulin resistance in Western Samoans: cross sectional study. BMJ 1996;313(7063):965-9.

34. Sorensen TI, Echwald S, Holm JC. Leptin in obesity. BMJ 1996;313(7063):953-4.

Endereço para correspondência:

Luís Henrique Canani

Serviço de Endocrinologia

Hospital de Clínicas de Porto Alegre

Rua Ramiro Barcelos 2350, prédio 12, 40 andar

90035-003 Porto Alegre, RS

Fax: (51) 2101-8777

E-mail: luiscanani@terra.com.br 